\section{Childhood amblyopia treatment: psychosocial implications for patients and primary carers}

\begin{abstract}
Purpose To investigate the psychosocial impact of amblyopia therapy on children and their carers.

Method The study was prospective and incorporated a repeated-measures design. A total of 59 carers were classified into occluded $(n=31)$ or nonoccluded group $(n=28)$. A

Carers felt temporarily more negative towards the child following onset of treatment with glasses. In this study, there is no evidence to indicate that occlusion therapy has negative psychosocial impact on carers and children alike.

Eye (2004) 18, 369-375. doi:10.1038/

sj.eye. 6700647
\end{abstract} questionnaire consisting of the Perceived Stress Index (PSI) and the Perceived Psychosocial Questionnaire (PPQ) was used to measure carer's perception of stress and psychosocial well-being of the child respectively prior to and following commencement of treatment. Parametric and nonparametric tests were used to analyse the data.

Results No significant difference in carer's stress $(P>0.05)$ and child's psychosocial wellbeing $(P>0.05)$ was observed between occluded and nonoccluded groups. Within occluded group, carer's stress $(P>0.05)$ and child's psychosocial well-being $(P>0.05) \mathrm{did}$ not differ significantly before and following commencement of treatment. Within the occluded group, carers felt more negative towards their child following onset of glasses therapy $(P<0.01)$ and became more positive when occlusion was introduced in the subsequent follow-up $(P<0.01)$.

Conclusions When compared to carers in the nonoccluded group, those with children undergoing occlusion therapy did not experience significantly more stress or perceived their child as exhibiting less psychosocial well-being. Within the occluded group, carers' stress level and child's psychosocial well-being did not significantly change following onset of occlusion therapy.
Keywords: amblyopia; psychosocial; occlusion; treatment; stress

\section{Introduction}

Amblyopia is an acquired defect in vision caused by abnormal visual experience early in life. On clinical testing, it is usually unilateral but may be bilateral. Amblyopia itself produces no apparent change in the appearance of ocular structures. The visual system is sensitive to the effects of abnormal visual experience only during a limited time in infancy and childhood when it is immature and plastic. This period extends from birth through the end of the first decade. Visual loss can be potentially reversed if amblyopia is detected and treated within this period. It is the most prevalent visual disability in children. A recent review estimated the prevalence of amblyopia in the UK to be between 2.4 and $6.1 \%$ in children of 3-4 years old. ${ }^{1}$

Numerous studies have documented the association between amblyopia and a variety of functional difficulties such as poor reading performance, ${ }^{2,3}$ and reduced stereopsis and motor abilities involving eye-hand coordination. ${ }^{4}$ As such functions are important for a child's development, 5,6 early detection and treatment of amblyopia is arguably the logical

YF Choong ${ }^{1}$, H Lukman², S Martin ${ }^{3}$ and DE Laws ${ }^{1}$

\author{
${ }^{1}$ Department of \\ Ophthalmology \\ Singleton Hospital \\ Swansea, UK \\ ${ }^{2}$ Centre for Psychology \\ University of Wales Institute \\ Cardiff, UK
}

${ }^{3}$ Department of Orthoptics Singleton Hospital

Swansea, UK

Correspondence:

YF Choong

5 Tarragon Way

Pontprennau

Cardiff CF23 8SN, UK

Tel: + 442920549788

Fax: + 442920416985

E-mail: yeefongchoong@ aol.com

Received: 17 December 2002

Accepted in revised form: 6 May 2003

The authors have no proprietary or financial interest in any products mentioned in the paper 
step forward. The need to implement treatment is more pressing when Tommila and Tarkkanen, ${ }^{7}$ and more recently Rahi et al, ${ }^{8,9}$ reported that the incidence of loss of vision in the healthy eye of individuals with amblyopia is higher compared to the normal population.

Currently, various screening procedures are used to identify amblyopia in children between the age of 3 and 4 years in the UK. ${ }^{10}$ Amblyopia is usually treated by correcting the refractive error with glasses and/or occlusion therapy involving patching of the nonamblyopic eye. Occlusion therapy has been used for over 250 years $^{11}$ and is aimed at encouraging the development of vision in the amblyopic eye. The efficacy of both the screening and treatment of amblyopia has been questioned recently by a systematic review. ${ }^{1}$ The authors concluded that there is insufficient evidence to indicate that amblyopia represents a significant disability that affects day-to-day functioning. They also highlighted a need for more investigations on the potential negative impact of treatment, particularly the psychosocial consequences of treatment on the child and his/her family. The objective of the present study is to address the latter point.

A few studies have begun to address the psychosocial impact of amblyopia and its treatment. Packwood et $a l^{12}$ surveyed 25 adults with amblyopia and reported that this sample perceived a greater degree of somatisation, obsessive-compulsive behaviour, interpersonal sensitivity, depression, and anxiety compared to those who has strabismus and those without amblyopia. In addition, a substantial number of those with amblyopia reported that amblyopia had interfered with school, work, and lifestyle. A few issues are worth noting. First of all, the small sample size and the retrospective nature of the study rendered the interpretation of the data difficult. It is unclear from the data if amblyopia per se had a direct effect on school, work, and lifestyle of those who had this condition. More importantly, the study did not specify in what ways amblyopia interfered with these factors and if the psychosocial impact of this condition was substantial enough to affect day-to-day functioning. Hence whether amblyopia can be considered a significant disability remains unclear. In addition, the psychosocial impact of the amblyopia treatment itself was not explored.

Recently, the Paediatric Eye Disease Investigator Group conducted a randomised clinical trial comparing the efficacy of occlusion therapy and atropine penalisation in children with moderate amblyopia. ${ }^{13,14}$ Part of the study aimed to evaluate the social stigma associated with the treatments. Approximately 5 weeks into treatment, parents of children with amblyopia given atropine treatment $(n=181)$ or occlusion therapy $(n=192)$ completed the 18-item Amblyopia Treatment
Index consisting of three subscales measuring adverse effects of treatment, difficulties with compliance, and social stigma of treatment. The findings indicated that parents of children undergoing occlusion therapy perceived significantly more problems with the treatment compared to those who were given atropine. However, both treatments were equally well tolerated. Although this study compared the adverse effects of two treatment modalities, it did not address the psychological impact of the treatments on the child and parents compared to the pretreatment period. Such information is needed to assess the treatment impact and determine if treatment benefits outweigh potential consequences.

The present study addresses three issues. The carer's perception of their personal stress level, psychosocial impact of occlusion therapy on the child, and carer-child relationship were evaluated prior to and following treatment.

\section{Materials and methods}

\section{Participants}

All primary carers attending a clinic in a large district general hospital, with children who were diagnosed with probable amblyopia or reduced vision, and about to undergo treatment (glasses and/or occlusion therapy) for the first time, were invited to participate in this research. This is an ongoing study that commenced since October 2000. To date, a total of 93 participants have been recruited. However, only 65 of them have completed the study. The data of these participants will be discussed in this paper.

\section{Demographics of carers}

Majority of the carers $(n=52)$ were mothers of the child seen in the clinic. A total of $96.9 \%$ of the sample was Caucasians. The mean age of carers was $32.4(\mathrm{SD}=6.3)$ years old. A total of $15.9 \%$ of the sample had tertiary education or higher; $38.1,42.9$, and $3.1 \%$ obtained college, secondary level, and primary level, respectively. In all, $80 \%$ of the sample had more than one child other than the one who was seen at the clinic. Among the siblings, majority (80\%) did not have amblyopia. The carer's understanding of amblyopia was also surveyed. A substantial number of them $(43.1 \%)$ admitted that they knew little or nothing about the condition.

\section{Demographics of amblyopic child}

There were 36 males and 29 females with a mean age of $57.1(\mathrm{SD}=20.9)$ months old (ie 4.8 years old). Of these children, $60 \%$ attended primary school; $21.5 \%$ were in 
nursery, $12.3 \%$ in play groups, and $6.2 \%$ cared at home. A total of $26(40.0 \%)$ were diagnosed anisometropic amblyopia, 24 (36.9\%) strabismic amblyopia, 13 (20.0\%) combined strabismic and anisometropic amblyopia, and two $(3.1 \%)$ refractive amblyopia. Mean visual acuity of the amblyopic eye at presentation was $0.32(\mathrm{SD}=0.54)$ using equivalent decimal Snellen notation.

\section{Materials}

Perceived Psychosocial Questionnaire Prior to the commencement of the study, a focus group was conducted to investigate the general issues related to amblyopia treatment, in particular occlusion therapy. The focus group consisted of ophthalmologists, orthoptists, parents of children who have experienced occlusion therapy, and a psychologist. The issues raised included the child's ability to concentrate, the degree of demands placed on the carer, the extent that the child cries more often, and play situations. These were incorporated into the development of the questionnaire used in this study, particularly the Perceived Psychosocial Questionnaire (PPQ). ${ }^{15} \mathrm{PPQ}$ is intended to measure the psychosocial well-being of the child. Higher overall PPQ score indicates lower psychosocial well-being.

Perceived Stress Index The stress level of carers was measured using the standardised global stress scale known as the Perceived Stress Index (PSI). ${ }^{16}$ This 10 -item index measures the degree to which general situations are perceived as stressful. Higher overall score represents a higher perception of stress experienced. It was important to measure general stress in this study as opposed to specific stress as a result of occlusion therapy. Occlusion therapy per se may not exert a direct impact on the carer's life, but may affect the predictability, controllability, and the degree of overload experienced by the carer in their daily lives. These three components have been repeatedly found to be important factors associated with stress and are measured by PSI. ${ }^{17,18}$

Carer-child relationship The carer-child relationship was depicted by how upset, irritated, patient, and attentive the carer felt towards the child. A total score was obtained by the summation of carer's response in these areas. Higher score indicates better relationship.

Questionnaire components In summary, the five subsections of the study questionnaire are listed in Table 1.
Table 1 Questionnaire components

Section 1: Background information on the carer and the child Section 2: Carer's knowledge on amblyopia and compliance to treatment

Section 3: Carer's perception of their stress level (PSI)

Section 4: Carer's perception of the child's psychological and social well-being (PPQ)

Section 5: Carer's relationship with the child and other family members

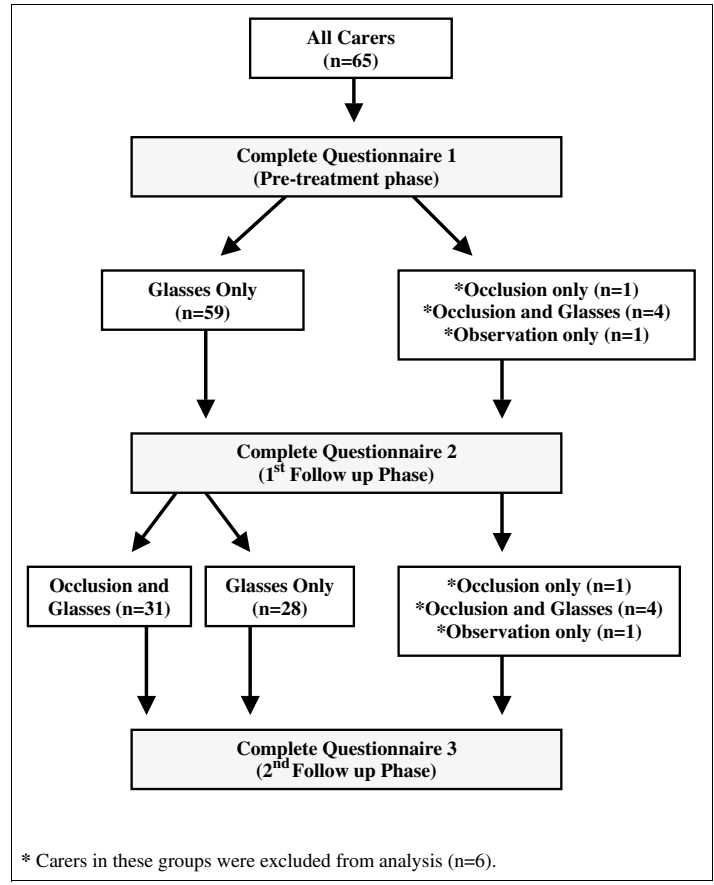

Figure 1 Study protocol flow chart.

\section{Study design}

The study was prospective and incorporated a repeatedmeasures design. Carers were asked to complete the previously discussed questionnaire at three different time points, that is, pretreatment phase, 1st follow-up phase, and 2nd follow-up phase (see Figure 1). The mean time interval between the pretreatment phase and 1st follow-up phase, and 1st follow-up phase and 2nd follow-up phase were $6.9(\mathrm{SD}=2.4)$ weeks and 7.4 $(\mathrm{SD}=8.6)$ weeks, respectively.

Pretreatment phase The diagnosis of amblyopia was confirmed in this first visit to the outpatient clinic. All carers were asked to complete one questionnaire prior to the commencement of amblyopia treatment. Following the consultation, carers were given instructions to proceed with the amblyopia treatment, that is, spectacle correction (glasses) and/or occlusion the next day. (Majority of patients with amblyopia initially received 
glasses. In the follow-up clinic, these patients were assessed for visual improvement in the amblyopic eye. When no significant improvement was shown, occlusion therapy was initiated. However, a small minority of patients commenced on occlusion therapy during the first (pretreatment) visit.) All except six children were prescribed glasses alone as their initial treatment. One of the six children was instructed to commence with occlusion therapy and four were treated with both occlusion and glasses. One was under observation only. The carers of these six children were excluded from the study to reduce bias.

1st follow-up phase All carers returned to the clinic for their child's routine follow-up, during which they were asked to complete the second questionnaire. This was carried out prior to their consultation. In this visit, some of the children who were initially prescribed glasses were placed on occlusion therapy $(n=31)$ because of the lack of improvement in the visual acuity of the amblyopic eye. The carers of these children were classified into the occluded group. Those who were not given occlusion therapy at this stage $(n=28)$ were classified into the nonoccluded group.

2nd follow-up phase During this visit, all participants completed the third questionnaire prior to seeing the orthoptists and/or ophthalmologist for further follow-up.

\section{Data analysis}

$t$-Tests were used to analyse the carer's perception of personal stress level, the child's psychosocial well-being, and carer-child relationship prior to and following the onset of occlusion therapy. The response of carers with children undergoing occlusion was also compared to those whose child was prescribed glasses only. Nonparametric tests were used to assess the separate components of the carer-child relationship. This was conducted for both between (using Mann-Whitney U-test) and within (using Wilcoxon signed rank test) the two groups. The data were analysed using SPSS version 10 (SPSS Inc.) and Excel version 7 (Microsoft

Corporation).

\section{Results}

\section{Carer's perceived stress}

There was no significant difference in PSI scores between occluded and nonoccluded groups $(p>0.05)$ (Table 2). Within the occluded group, carer's perception of stress
Table 2 Mean PSI scores of carers in occluded and nonoccluded groups (standard deviations are in parentheses)

\begin{tabular}{lccc}
\hline Phase & $\begin{array}{c}\text { Occluded } \\
\text { group }\end{array}$ & $\begin{array}{c}\text { Nonoccluded } \\
\text { group }\end{array}$ & $\begin{array}{c}\text { Between-groups } \\
\text { p-value }\end{array}$ \\
\hline Pretreatment & $13.27(5.30)$ & $13.52(6.58)$ & $>0.05$ \\
1st follow-up & $13.42(5.46)$ & $12.81(5.46)$ & $>0.05$ \\
2nd follow-up & $12.47(6.74)$ & $13.25(6.29)$ & $>0.05$ \\
Within-group $p$-value & $>0.05$ & $>0.05$ & \\
\hline
\end{tabular}

Table 3 Mean PPQ scores of carers in occluded and nonoccluded groups (standard deviations are in parentheses)

\begin{tabular}{lccc}
\hline Phase & $\begin{array}{c}\text { Occluded } \\
\text { group }\end{array}$ & $\begin{array}{c}\text { Nonoccluded } \\
\text { group }\end{array}$ & $\begin{array}{c}\text { Between-groups } \\
\text { p-value }\end{array}$ \\
\hline Pretreatment & $23.51(9.38)$ & $20.12(7.99)$ & $>0.05$ \\
1st follow-up & $22.53(10.44)$ & $19.33(7.12)$ & $>0.05$ \\
2nd follow-up & $23.81(10.44)$ & $20.36(9.46)$ & $>0.05$ \\
Within group $p$-value $>0.05$ & $>0.05$ & \\
\hline
\end{tabular}

did not differ significantly between the pretreatment, 1st follow-up, and the 2nd follow-up phases $(p>0.05)$. The result was similar within the nonoccluded group.

\section{Carer's perception of child's psychosocial well-being}

The PPQ scores between the occluded group and nonoccluded group did not differ significantly between the three phases $(p>0.05)$. However, the occluded group scored consistently higher in all three phases. No significant difference in PPQ scores was observed within occluded $(p>0.05)$ and nonoccluded groups $(p>0.05)$ (Table 3).

\section{Relationship between carer and child}

The carer-child relationship scores were similar between the occluded and nonoccluded groups (Table 4). There were no significant between-group differences in how upset, irritated, patient, or attentive carers were towards the child across all three phases $(p>0.05)$ (Table 4$)$.

It is interesting to note that the carer-child relationship score was lower for both groups in 1st follow-up phase compared to the pretreatment phase (Tables 5 and 6). In particular, they were more upset and irritated with the child during this phase. However, these negative feelings were reduced in the 2nd follow-up phase. These findings only reached statistical significance within the occluded group $(p<0.01)$. Carers in this group were significantly less upset and irritated during 2nd follow-up phase compared to the 1st follow-up phase (Table 5). A similar pattern emerged within the nonoccluded group, but the 
difference was not substantial enough in most cases to produce statistical significance with the exception of carer's irritability towards the child. Carers in the nonoccluded group experienced a significant reduction in their irritable feelings towards the child $(p<0.05)$ in the 2nd follow-up phase compared to the 1st follow-up phase (Table 6). In both groups, carers were as patient and attentive to the child in all three phases.

\section{Effect of glasses on children}

The finding that both groups experienced a lower carerchild relationship score during the 1st follow-up phase indicated the possibility that glasses per se may be the reason for this phenomenon. All patients in our sample were prescribed glasses following their pretreatment phase to correct the refractive error. Hence glasses alone may influence the well-being of the carer and/or the child. An additional analysis was conducted on carers who have children given glasses as their initial treatment

Table 4 Mean score of carer-child relationship (standard deviations are in parentheses)

\begin{tabular}{lccc}
\hline Phase & $\begin{array}{c}\text { Occluded } \\
\text { group }\end{array}$ & $\begin{array}{c}\text { Nonoccluded } \\
\text { group }\end{array}$ & $\begin{array}{c}\text { Between-groups } \\
\text { p-value }\end{array}$ \\
\hline Pretreatment & $22.87(3.38)$ & $22.30(3.93)$ & $>0.05$ \\
1st follow-up & $20.19(5.10)$ & $20.93(5.37)$ & $>0.05$ \\
2nd follow-up & $23.06(3.21)$ & $22.68(4.10)$ & $>0.05$ \\
\hline
\end{tabular}

during the 1st follow-up phase $(n=59)$. Carer's perception of his/her stress level, child's psychosocial well-being, and carer-child relationship between pretreatment and 1st follow-up phase were examined. Results indicated no difference in PSI $(p>0.05)$ and PPQ $(p>0.05)$ scores between these phases. However, carerchild relationship score was significantly lower following the onset of glasses in the 1st follow-up phase $(p<0.01)$ (Table 7). Particularly, carers were significantly more upset $(p<0.01)$ and irritated $(p<0.01)$ with the child at this phase compared to the pretreatment phase.

\section{Discussion}

The findings of this study have highlighted three important points. Firstly, the study demonstrated that carers with children undergoing occlusion therapy and those prescribed glasses only did not differ significantly in their experience of stress. The implication of this finding suggests that with regard to carers, occlusion therapy is not more stressful than glasses. One study reported that occlusion therapy has more problems with adverse effects, compliance, and social stigma compared to atropine treatment. ${ }^{13}$ This suggests that occlusion therapy may be a more stressful treatment compared to atropine. However, whether this was the case was not addressed in that study. Our study showed that when compared to glasses, occlusion therapy was not a more stressful treatment for the carer. Additionally, carer's

Table 5 Mean score of carer-child relationship, how upset and irritated carers in occluded group were with the child across all phases (standard deviations are in parentheses)

\begin{tabular}{lrrrrc}
\hline & Pretreatment & 1st follow-up & 2nd follow-up & $\begin{array}{c}\text { Between pretreatment and } \\
\text { 1st follow-up phases p-value }\end{array}$ & $\begin{array}{c}\text { Between 1st follow-up and } \\
\text { 2nd follow-up p-value }\end{array}$ \\
\hline Carer-child relationship & $22.87(3.38)$ & $20.19(5.10)$ & $23.06(3.21)$ & $<0.01$ & $<0.01$ \\
Upset & $5.67(1.69)$ & $4.43(2.24)$ & $5.90(1.08)$ & $0.039(\mathrm{~ns})^{\mathrm{a}}$ & $<0.01$ \\
Irritated & $5.53(1.53)$ & $4.40(2.33)$ & $5.97(1.19)$ & $0.027(\mathrm{~ns})^{\mathrm{a}}$ & $<0.01$ \\
Patient & $5.77(1.36)$ & $5.43(1.65)$ & $5.55(1.73)$ & $>0.05(\mathrm{~ns})$ & $>0.05(\mathrm{~ns})$ \\
Attentive & $5.90(1.12)$ & $5.77(1.04)$ & $5.65(1.71)$ & $>0.05(\mathrm{~ns})$ & $>0.05(\mathrm{~ns})$ \\
\hline
\end{tabular}

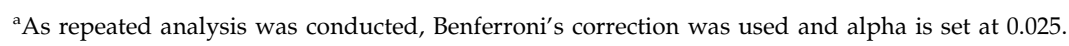

ns=nonsignificant.

Table 6 Mean score of carer-child relationship, how upset and irritated carers in nonoccluded group were with the child across the phases (standard deviations are in parentheses)

\begin{tabular}{|c|c|c|c|c|c|}
\hline & Pretreatment & 1st follow-up & 2nd follow-up & $\begin{array}{l}\text { Between pretreatment and } \\
\text { 1st follow-up phases } \mathrm{p} \text {-value }\end{array}$ & $\begin{array}{l}\text { Between 1st follow-up and } \\
\text { 2nd follow-up } \mathrm{p} \text {-value }\end{array}$ \\
\hline Carer-child relationship & $22.30(3.93)$ & $20.93(5.37)$ & $22.68(4.10)$ & $>0.05$ (ns) & $>0.05 \quad(\mathrm{~ns})$ \\
\hline Upset & $5.61(1.34)$ & $4.86(2.07)$ & $5.86(1.33)$ & $>0.05$ (ns) & $0.038(\mathrm{~ns})^{\mathrm{a}}$ \\
\hline Irritated & $5.43(1.45)$ & $4.61(2.20)$ & $5.68(1.54)$ & $>0.05$ (ns) & $<0.01$ \\
\hline Patient & $5.63(1.33)$ & $5.50(1.64)$ & $5.59(1.42)$ & $>0.05$ (ns) & $>0.05 \quad$ (ns) \\
\hline Attentive & $5.68(1.39)$ & $5.96(1.26)$ & $5.71(1.41)$ & $>0.05$ (ns) & $>0.05 \quad$ (ns) \\
\hline
\end{tabular}

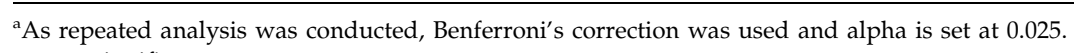

$\mathrm{ns}=$ nonsignificant. 
Table 7 Mean score of how upset and irritated carers with children prescribed glasses only (standard deviations are in parentheses)

\begin{tabular}{lccc}
\hline & Pretreatment & 1st follow-up & $\begin{array}{c}\text { Between } \\
\text { phases p-value }\end{array}$ \\
\hline $\begin{array}{l}\text { Carer-child } \\
\text { relationship score }\end{array}$ & $22.60(3.63)$ & $20.54(5.20)$ & $<0.01$ \\
$\begin{array}{l}\text { Upset with child } \\
\text { Irritated with child }\end{array}$ & $5.64(1.52)$ & $4.66(2.14)$ & $<0.01$ \\
\hline
\end{tabular}

experience of general stress before and following commencement of treatment did not differ significantly. This implies that carer's state of general stress was not influenced substantially by the treatment. This finding appears to contradict the conventional anecdotal evidence that suggests occlusion therapy to be a stressful procedure for carers. There are a few plausible explanations for this discrepancy. Firstly, occlusion therapy itself may represent a stressful experience, but the degree of stress is not substantial enough to exert a measurable impact. It is also possible that the stress experienced is transient. Amblyopia treatment may have specific difficulties in the initial stages of treatment when acuity is at its worst, but subsequently becomes more acceptable as the visual acuity improves. Therefore, carers may experience significant stress at onset of treatment, but their level of stress reduces at the time of measurement. Finally, there are few individuals who may be very susceptible to stress, and the clinicians' experience with these individuals may have biased their impression, whereas the majority of carers cope well.

The second point concerns the psychosocial impact of occlusion treatment on the amblyopic child. A common complaint from parents with children undergoing occlusion therapy is that the child becomes more distressed following the onset of treatment. The repeated application of a patch for occlusion therapy may cause skin irritation or rarely an allergic reaction. The occlusion of the nonamblyopic eye understandably causes a degree of distress in a young child. Although occlusion therapy can be distressing, our data did not indicate that children with occlusion were experiencing significantly more distress following the onset of treatment. In addition, carers in the occluded group did not perceive their child to experience poorer psychosocial well-being as compared to those just given glasses. Our findings seem to suggest that occlusion therapy does not exert a significant adverse psychosocial impact on children undergoing occlusion and that it is not more distressing than treatment with glasses.

Thirdly, with regard to the carer-child relationship, it is interesting to note that carers felt that they are more upset and irritated with the child following the onset of treatment with glasses. This experience was reversed and carers felt less upset and irritated during the 2nd followup phase when treatment has progressed for a time. These findings were more marked among carers within the occluded group. The onset of occlusion was not related with more negative experience but rather surprisingly, carers in the occluded group experienced significantly more positive feelings towards the child as opposed to carers in the nonoccluded group. It appears that carers with children undergoing occlusion therapy were more understanding towards their child's treatment experience. Since further analysis showed that glasses alone also have the same impact, it is plausible that prescribing glasses as treatment exerts a negative but transient influence on the carer-child relationship. The addition of occlusion therapy to glasses did not contribute to a substantial worsening in the carer-child relationship. Hence, our data support the findings of the Paediatric Eye Disease Investigator Group that occlusion therapy is generally well tolerated. ${ }^{13}$

There are a few limitations in this study that should be taken into consideration when interpreting our findings. Firstly, the period between consultations or study phases differs between each participant. This could possibly influence the results. For example, carers who had a longer period between the pretreatment phase and the 1st follow-up phase may have a different response compared to those who had a shorter period. This limitation should be noted, although it is practically difficult to control the length of intervals between followup in clinical settings. Secondly, our study only measures the perception rather than the objective measure of carer's stress, child's psychosocial well-being, and carer-child relationship. Hence we can only comment on the perceived experience of the carer rather than what is actually experienced. It is important that future studies evaluate the objective experience of amblyopia treatment in patients and carers alike. Thirdly, there are various factors such as treatment compliance, treatment acceptability, duration of occlusion, density of amblyopia, types of amblyopia, and others that may influence the success of amblyopia treatment. ${ }^{19-24}$ These in turn may have a significant influence on the psychosocial impact of amblyopia treatment. Data are currently being collected for further analysis in this on going study that will address some of these issues.

In summary, our study has attempted to address the psychosocial impact of occlusion therapy on patient and carer. Following onset of occlusion therapy, carers did not perceive their stress level to be higher or their child's psychosocial well-being to be poorer. However, carers were temporarily more upset and irritated with the child following onset of amblyopia treatment with glasses. The transient nature of this experience is reassuring. In 
conclusion, there is at present no evidence from this study to suggest that occlusion therapy exerts a significant adverse psychosocial impact on carers and patients alike. This information should be taken into account when discussing the advantages and disadvantages of amblyopia treatment and in particular that of occlusion therapy.

\section{Acknowledgements}

This study is funded by a grant from the Welsh Office for Research and Development (WORD) and was presented in part at the Annual Congress, The Royal College of Ophthalmologists, Manchester, May 2002 and the Association for Research in Vision and Ophthalmology (ARVO), Fort Lauderdale, Florida, May 2002.

\section{References}

1 Snowdon S, Stewart-Brown SL. Pre-school Vision Screening: Results of a Systematic Review. University of York: NHS Centre for Reviews and Dissemination, 1997.

2 Grosvenor T. Are visual anomalies related to reading ability? J Am Optom Assoc 1977; 48(4): 510-517.

3 Grisham JD, Simons HD. Refractive error and the reading process: a literature analysis. J Am Optom Assoc 1986; 57(1): $44-55$.

4 McGee RWS, Simpson A, Silva PA. Stereoscopic vision and motor ability in a large sample of seven-year-old children. J Hum Movement Stud 1991; 13: 343-352.

5 Williams SM, Sanderson GF, Share DL, Silva PA. Refractive error, IQ and reading ability: a longitudinal study from age seven to 11. Dev Med Child Neurol 1988; 30(6): 735-742.

6 Fielder AR, Moseley MJ. Does stereopsis matter in humans? Eye 1996; 10 (Part 2): 233-238.

7 Tommila V, Tarkkanen A. Incidence of loss of vision in the healthy eye in amblyopia. Br J Ophthalmol 1981; 65(8): 575-577.

8 Rahi JS, Logan S, Borja MC, Timms C, Russell-Eggitt I, Taylor D. Prediction of improved vision in the amblyopic eye after visual loss in the non-amblyopic eye. Lancet 2002; 360(9333): 621-622.

9 Rahi J, Logan S, Timms C, Russell-Eggitt I, Taylor D. Risk, causes, and outcomes of visual impairment after loss of vision in the non-amblyopic eye: a population-based study. Lancet 2002; 360(9333): 597-602.

10 Stewart-Brown SL, Haslum MN, Howlett B. Preschool vision screening: a service in need of rationalisation. Arch Dis Child 1988; 63(4): 356-359.

11 de Buffon M. Dissertation sur la cause de strabisme ou de les yeux louches. Bull Mem Acad Sci 1743; 3: 174.

12 Packwood EA, Cruz OA, Rychwalski PJ, Keech RV. The psychosocial effects of amblyopia study. J AAPOS 1999; 3(1): 15-17.

13 The study was conducted by the Pediatric Eye Disease Investigation Group (PEDIG). A randomized trial of atropine $v$ s patching for treatment of moderate amblyopia in children. Arch Ophthalmol 2002; 120(3): 268-278.

14 Group TPEDI. The clinical profile of moderate amblyopia in children younger than 7 years. Arch Ophthalmol 2002; 120(3): 281-287.

15 Lukman H, Choong YF, Martin S, Laws DE. Development of Perceived Psychosocial Questionnaire (PPQ), a psychosocial measure to study the effect of amblyopia treatment. Paper presented at The Association of Research in Vision and Ophthalmology (ARVO), 2002, Florida, USA.

16 Cohen S, Kamarck T, Mermelstein R. A global measure of perceived stress. J Health Soc Behav 1983; 24(4): 385-396.

17 Friend TH. Behavioral aspects of stress. J Dairy Sci 1991; 74(1): 292-303.

18 Steptoe A. Invited review. The links between stress and illness. I Psychosom Res 1991; 35(6): 633-644.

19 Smith LK, Thompson JR, Woodruff G, Hiscox F. Factors affecting treatment compliance in amblyopia. J Pediatr Ophthalmol Strabismus 1995; 32(2): 98-101.

20 Fielder AR, Irwin M, Auld R, Cocker KD, Jones HS, Moseley MJ. Compliance in amblyopia therapy: objective monitoring of occlusion. Br J Ophthalmol 1995; 79(6): 585-589.

21 Woodruff G, Hiscox F, Thompson JR, Smith LK. Factors affecting the outcome of children treated for amblyopia. Eye 1994; 8 (Part 6): 627-631.

22 Fielder AR, Auld R, Irwin M, Cocker KD, Jones HS, Moseley MJ. Compliance monitoring in amblyopia therapy. Lancet 1994; 343(8896): 547.

23 Newsham D. A randomised controlled trial of written information: the effect on parental non-concordance with occlusion therapy. $\mathrm{Br} J$ Ophthalmol 2002; 86(7): 787-791.

24 Newsham D. Parental non-concordance with occlusion therapy. Br J Ophthalmol 2000; 84(9): 957-962. 